\title{
DE TODO UN POCO
}

La excepcional situación provocada por la COVID-19 durante este año 2020, ha obligado a suspender o modificar muchas de las actividades en las que de forma habitual participa la Facultad de Ciencias Biológicas y Ambientales (FCCBA) de la Universidad de León (ULe). A pesar de ello, y en la medida de lo posible, hemos continuado colaborando y organizando eventos tanto de carácter científico y cultural como formativo y divulgativo.

\section{Jornadas, congresos y conferencias}

Con motivo de la conmemoración del Día Internacional de la mujer y la niña en la Ciencia (Fig. 1), y con la finalidad visibilizar el trabajo de las científicas, se programaron desde el Vicerrectorado de Investigación y Transferencia de la ULe, charlas, talleres y mesas redondas en centros universitarios y en diversos colegios e institutos de la provincia de León. Las actividades se desarrollaron durante la primera quincena el mes de febrero, iniciándose el día 3 en el Aula Magna de la Facultad con la conferencia titulada 'Tras los pasos de las pioneras en ciencia. El techo de cristal". La charla fue impartida por las investigadoras Marta Lombó, Alba Manga y Alba María García Lino. Otras 14 profesoras einvestigadoras de la FCCBA participaron en diferentes talleres sobre: Biología del Desarrollo, Seres vivos, Tecnología de los Alimentos, Fisiología y Biotecnología de las Plantas y Fisiología Animal.

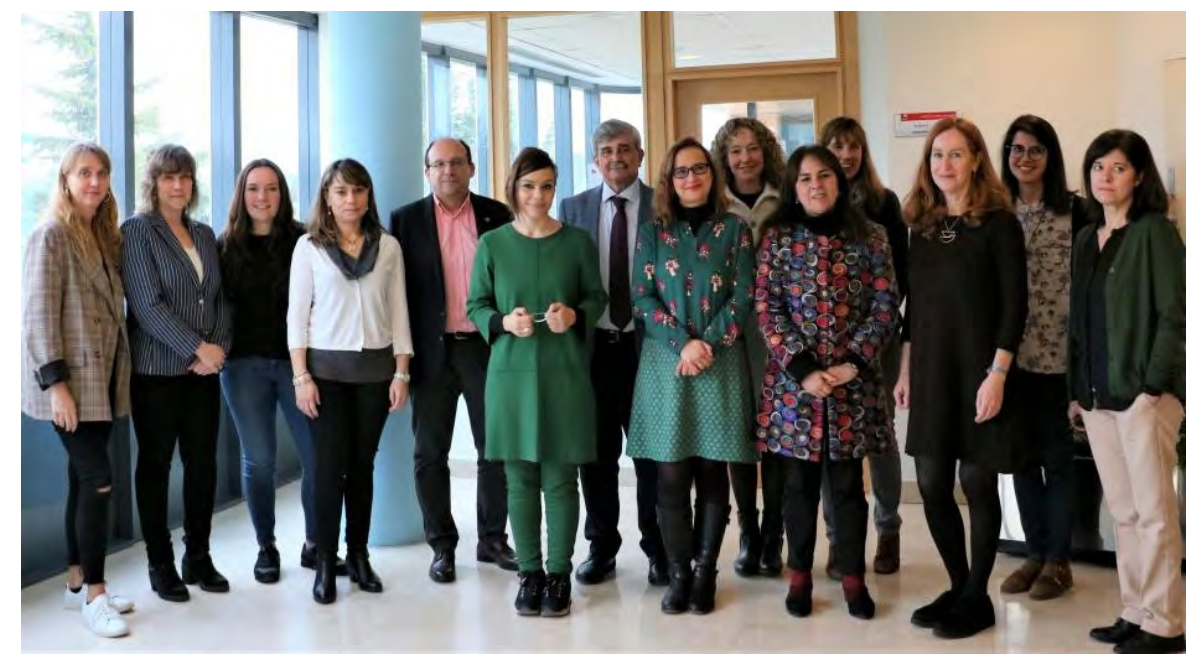

Figura 1. Representación de investigadoras y científicas participantes en el Día Internacional de la mujer y la niña en la Ciencia acompañadas del Rector, el Vicerrector de Investigación y Transferencia y la Vicerrectora de Relaciones Institucionales y con la Sociedad de la ULe

Cabe destacar también la exitosa acogida de la segunda edición de $\boldsymbol{E x}$ pociencia Unileon 2020 (Fig. 2) celebrada los días 12 y 13 de febrero en Pon- 
ferrada y 18, 19y 20 del mismo mes en León. Expociencia Unileon es un evento de divulgación científica organizado por la ULe en colaboración con la Fundación Española para la Ciencia y la Tecnología (FECYT) que trata de fomentar la vocación investigadora y emprendedora y difundir la labor que desempeñan los grupos de investigación. Más de treinta grupos de investigación de la ULe participaron en el evento, organizando talleres y ponencias a los que asistieron 8000 visitantes y un total de 4500 alumnos pertenecientes a 55 centros educativos de la provincia. Numerosos profesores e investigadores de nuestra Facultad, pertenecientes a siete grupos de investigación, participaron en este evento con las actividades siguientes: "Taller de Experimentos Fascinantes con Plantas", "Análisis atmosférico de aerosoles y bioaerosoles y sus interrelaciones", "Biominerales en acción. Cómo fabricamos y qué superpoderes nos confieren los biominerales", "Las "movidas" de las plantas: ¿cómo se defienden frente a los cambios?", "Botánico por un día: 2020 Vigilando la biodiversidad", "Si tú me dices Química, lo dejo todo" y "¿Hay vida después de un incendio?: La ciencia de la Ecología responde".
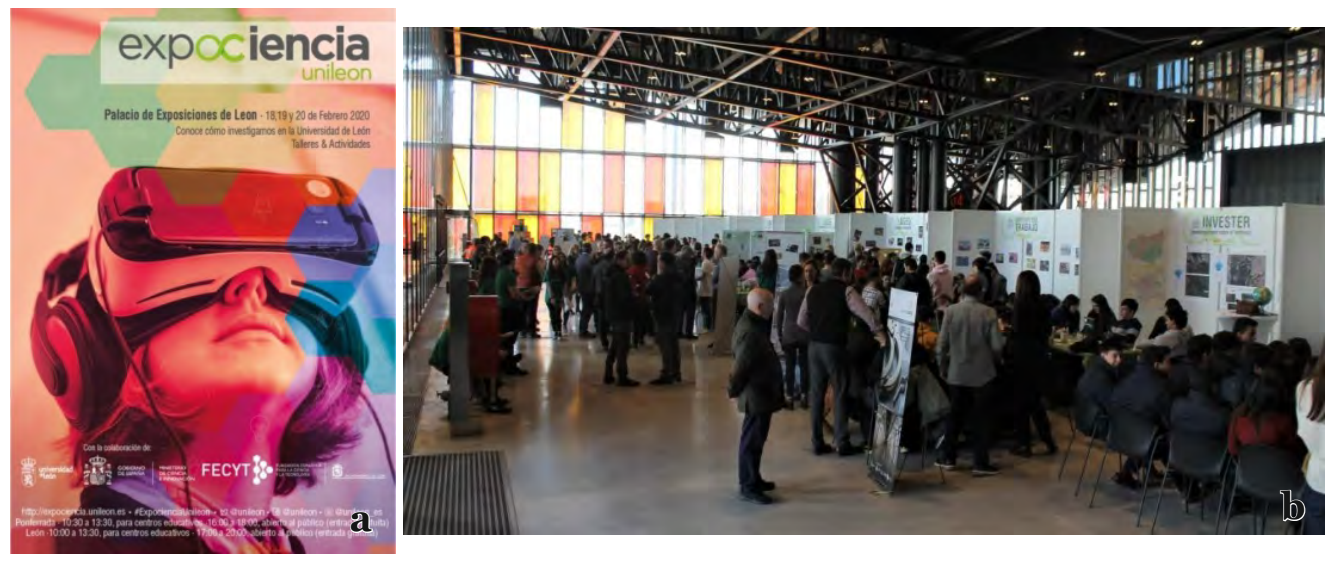

Figura 2. a) Cartel informativo Expociencia Unileon 2020; b) Palacio de Congresos de León donde se desarrolló la Expociencia

Durante los días 20 a 22 de febrero se celebraron en la Universidad de Trás-os-Montes e Alto Douro (UTAD) (Portugal) las II Jornadas Ibéricas de Genética y Biotecnología (Fig. 3), organizadas por la Dra. M. ${ }^{a}$ Luz Centeno en colaboración con profesores de las áreas de Fisiología Vegetal y de Genética, el Equipo Decanal, la Asociación de Biotecnólogos de León (ABLE) y profesores de la UTAD. Asistieron a las Jornadas cerca de 200 participantes con presencia de investigadores de reconocido prestigio en los campos de la Genética y Biotecnología vegetal, humana, animal y de microrganismos. Las contribuciones científicas se presentaron en sesiones de pósteres, comunicaciones orales y conferencias. 


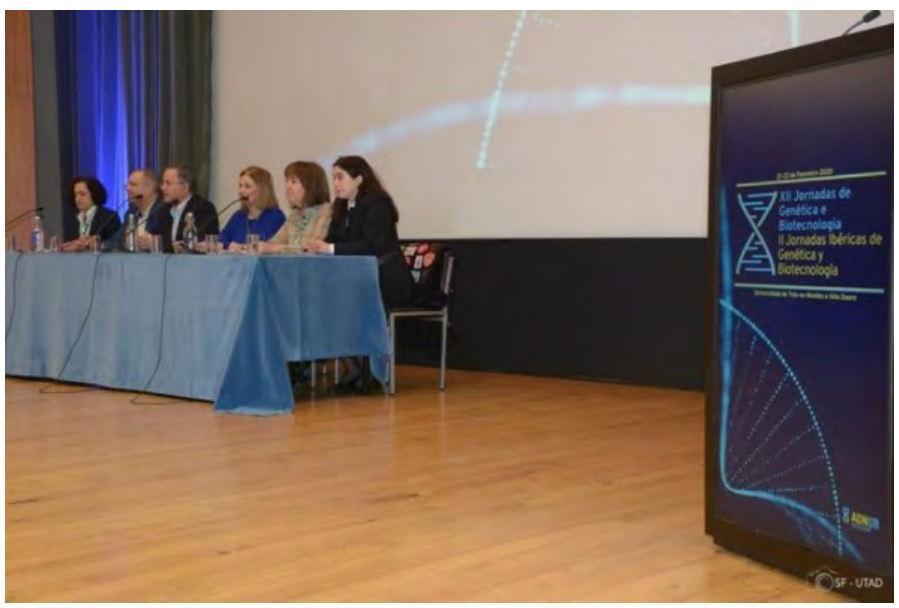

Figura 3. Celebración de las II Jornadas Ibéricas de Genética y Biotecnología en la Universidad de Trás-os-Montes e Alto Douro (UTAD) (Portugal)

Como en años anteriores y con el objetivo de acercar la ciencia a los diferentes sectores de la sociedad leonesa, ABLE organizó, entre el 6 de marzo y el 3 de abril, el ciclo de conferencias de divulgación científica ConCiencia en la Fundación Sierra Pambley. La situación provocada por la COVID-19 obligó a la cancelación de la actividad, pudiendo impartirse solamente la ponencia titulada "Bichos sencillos, extraños, peligrosos, ¿feos? ..., pero útiles" por parte del Decano de nuestra Facultad, el Dr. Antonio Laborda Navia.

Los días 11, 12 y 13 de mayo se celebró la primera edición de las Jornadas de Puertas Abiertas on line, organizadas por los Vicerrectorados de Estudiantes y Relaciones Institucionales y con la Sociedad de la Universidad de León. Bajo el título "La Universidad se acerca a ti" se ofrecieron charlas informativas en directo de profesores y expertos, visitas virtuales y sesiones de chat en las que se trasladó información sobre el desarrollo de la EBAU, motivaciones y razones por las que estudiar en la ULe, oferta de Grados, etc. En esta misma línea y con el mismo formato, los días 16, 17 y 18 de junio tuvieron lugar las I Jornadas online de Posgrados de la $\boldsymbol{U} \boldsymbol{L E}$ en las que se ofreció información sobre la oferta de Másteres y Programas de Doctorado que se imparten en los Campus de León y Ponferrada.

La asociación ABLE organizó, durante un año más, y en colaboración con la Federación Española de Biotecnólogos (FEBBiotec), el ciclo de charlas de divulgación científica Con Ciencia, Té que fueron retransmitidas durante todos los jueves del mes de diciembre a través de la plataforma IGTV de la red social Instagram. Las charlas fueron impartidas por investigadores predoctorales y alumnos de Máster y Grado y los títulos de las mismas fueron: "Superbacterias, ¿el enemigo del futuro o del presente?", "Necesidades ambientales en gatos: cómo hacerlos más felices", "Bdellovibrio bacteriovorus ¿Una solución a las multirresistencias?”, “Mixtamalización y otras alcalinizaciones. Aplicaciones culinarias e industriales del pasado, presente y futuro", "Las plantas más jetas: 
transferencia horizontal en plantas parásitas", "Esta masa... iise sale de madre!!” " El silicio en microprocesadores ¿Tiene sustitutos?”.

\section{Cursos y talleres}

Durante los días 10 y 11 de febrero se celebró la segunda edición del curso de Alcoholes, organizada por la Asociación ABLE. Aitor Balsameda (investigador predoctoral de la Universidad Rovira i Virgili) explicó los aspectos básicos de los procesos fermentativos comunes de bebidas fermentadas como el vino, la cerveza y la sidra: microorganismos, rutas metabólicas y materias primas.

Como ya viene siendo habitual, profesores de la Facultad participaron también durante este año en la dirección e impartición de Cursos de Verano y de Extensión Universitaria. Entre ellos pueden destacarse: "Micología Aplicada”, bajo la dirección de la Dra. Ana Belén Fernández Salegui y "El futuro de los incendios forestales: nuevas tecnologías y territorios resilientes" dirigido por los Dres. Alfonso Fernández Manso y Leonor Calvo Galván.

\section{Innovación docente y otras actividades}

El PDI de la Facultad de Ciencias Biológicas y Ambientales continúa implicado de forma activa con la innovación docente. Por ejemplo, en el II Congreso Internacional de Innovación Docente e Investigación en Educación Superior: Avanzando en las Áreas, celebrado en Madrid los días 11, 12 y 13 de noviembre, el simposio: "Incentivar el interés por materias Stem en niveles universitarios y pre-universitarios mediante Metodologías $A a+(A b p$, Aps)" fue coordinado por la Dra. Carmen Acedo y contó con cinco ponencias presentadas por otras tantas profesoras de nuestra Facultad.

En lo que a otras actividades respecta, el día 13 de febrero se celebró la segunda parte del proyecto Biotechnofarm organizado por la Federación Española de Biotecnólogos (FEBiotec). El proyecto pretende acercar la Biotecnología a las escuelas y está orientado a estudiantes de $4^{\circ}$ de E.S.O. y de Bachillerato. 15 alumnos de seis centros educativos participaron este año en diferentes talleres científicos en los laboratorios de la Facultad.

62 niños de entre 6 y 12 años (hijos y familiares de empleados de la ULe) participaron durante los días 24, 25 y 26 de febrero en el programa ConciliaULe (Fig. 4) organizado por el Área Social del Vicerrectorado de Responsabilidad Social, Cultura y Deportes de la ULe. El objetivo de este programa es atender a la demanda de los trabajadores y favorecer así la conciliación laboral, familiar y personal del personal de la ULe. Los niños realizaron un recorrido por cuatro centros del Campus de Vegazana, entre ellos la FCCBA donde realizaron distintos talleres y actividades. 


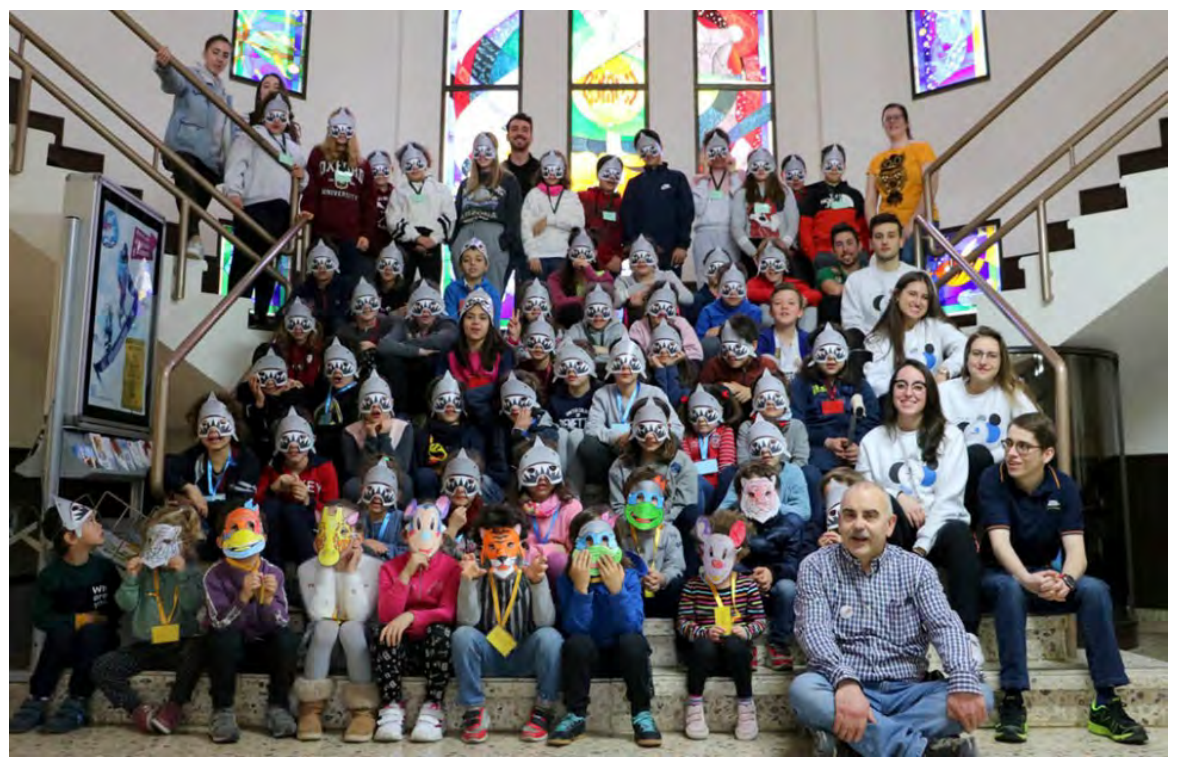

Figura 4. Participantes en el programa Conciencia-ULe 2020 en la entrada principal de la FCCBA

La pandemia no impidió la celebración de Geolodía 2020 en el mes de mayo. Para esta excepcional ocasión y debido a la imposibilidad de asistir de forma presencial a las excursiones, los organizadores prepararon guías de campo, explicaciones de las rutas en vídeo, fotografías y gráficos del paraje de 'Las Torcas de Barrientos' a través de los cuales se pudieron realizar de forma virtual las salidas de campo programadas y así descubrir toda la geología que se muestra en ellas. La actividad fue organizada por la Sociedad Geológica de España (SGE) con el apoyo, entre otras instituciones, de la Universidad de León a través del Grupo 'Q-Geo' que coordina la Dra. Esperanza Fernández.

También durante el periodo de confinamiento se desarrolló desde la Facultad la actividad Yo me quedo en casa estudiando Fisiología Vegetal, coordinada por la Dra. Penélope García Angulo. Los alumnos de $2^{0}$ curso del Grado en Biotecnología elaboraron una serie de vídeos de divulgación científica de corta duración (3-4 minutos) en los que presentaron pequeñas píldoras de información sobre temas que forman parte de los contenidos que estaban cursando (Fig. 5). Todos los vídeos están disponibles en la web de la ULe.

Las plantas, placas solares naturales.

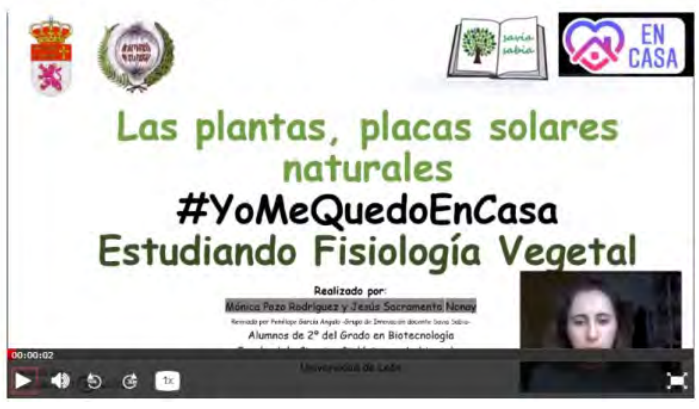

Figura 5. Ejemplo de vídeo elaborado por los alumnos de $2^{\circ}$ de Biotecnología en la asignatura de Fisiología Vegetal 
Se celebraron también las VII Jornadas de Prácticas de Gestión de Flora, organizadas por la Dra. Carmen Acedo. Las Jornadas se incluyen como una actividad en la programación formativa de la asignatura del Grado en Ciencias Ambientales "Gestión de Flora". Este año, y debido a la situación provocada por la pandemia, se organizó una sala de exposición virtual para que los estudiantes presentaran los paneles con los resultados de los proyectos prácticos realizados durante el semestre.

Del 8 de octubre al 13 de noviembre se presentó en la Sala de Exposiciones de la Facultad la Exposición de Fotografia "Esencialmente Invisible" de la artista vallisoletana Nuria Sancho. La exposición incluía retratos que nos invitaban a conectar con nosotros mismos y la belleza que nos rodea.

Del 9 al 15 de noviembre tuvo lugar la XVII edición de la Semana de la Ciencia en Castilla y León con la coordinación del Parque Científico Universidad de Valladolid y la colaboración de la Consejería de Educación a través de la Fundación Universidades y Enseñanzas Superiores de Castilla y León (Fig. 6). Cabe destacar, entre las actividades programadas por la Universidad de León, la charla impartida por la Dra. Leonor Calvo titulada "Problemática de los incendiosforestales".

Los días 11 y 12 de noviembre tuvieron lugar las Jornadas de Investigadoras de Castilla y León en su VI edición, organizadas por las cuatro Universidades de Castilla y León. La Dra. Carmen Marín formó parte del Comité Organizador de las mismas y la Dra. María Paz Herráez impartió la conferencia titulada "El legado masculino ¿quéheredamos de papá?"

La tarde del 27 de noviembre se celebró la segunda edición de la "Noche europeadel@s investigador@s”. El programa está compuesto por microcharlas, conferencias y talleres divulgativos para todos los públicos impartidos por investigadores de la Universidad de León. Profesores de la Facultad participaron en el evento organizando una visita virtual al Herbario Jaime Andrés -LEB-de la Universidad de León a través del canal YouTube de la Ule.

Correspondería en este punto comentar la celebración de la fiesta en honor a nuestro patrón San Alberto Magno, que lamentablemente ha tenido que posponerse hasta que la situación sanitaria lo permita. Esperemos que pronto podamos retomar su conmemoración, así como la celebración, sin ningún tipo de limitación, de las numerosas actividades en las que participa nuestra Facultad. 